\title{
Dual-band Frequency Selective Surface Bandpass Filters in Terahertz Band
}

\author{
Limei $\mathrm{Qi}^{1}{ }^{*}$ and $\mathrm{Chao} \mathrm{Li}^{2}$ \\ ${ }^{1}$ School of Electronic Engineering, Beijing University of Posts and Communications, Beijing 100876, China \\ ${ }^{2}$ Key Laboratory of Electromagnetic Radiation and Sensing Technology, Chinese Academy of Sciences, \\ Beijing 100190, China
}

(Received September 16, 2015 : revised November 2, 2015 : accepted November 24, 2015)

\begin{abstract}
Terahertz dual-band frequency selective surface filters made by perforating two different rectangular holes in molybdenum have been designed, fabricated and measured. Physical mechanisms of the dual-band resonant responses are clarified by three differently configured filters and the electric field distribution diagrams. The design process is straightforward and simple according to the physical concept and some formulas. Due to the weak coupling between the two neighboring rectangle holes with different sizes in the unit cell, good dual-band frequency selectivity performance can be easily achieved both in the lower and higher bands by tuning dimensions of the two rectangular holes. Three samples are fabricated, and their dual-band characteristics have been demonstrated by a $\mathrm{THz}$ time-domain spectroscopy system. Different from most commonly used metal-dielectric structure or metal-dielectric-metal sandwiched filters, the designed dual-band filters have advantages of easy fabrication and low cost, the encouraging results afforded by these filters could find their applications in dual-band sensors, $\mathrm{THz}$ communication systems and other emerging $\mathrm{THz}$ technologies.
\end{abstract}

Keywords : Metamaterial filter, Band-pass, Frequency selective surface, Multi-band filter OCIS codes : (160.3918) Metamaterials; (050.6624) Sub-wavelength structures; (260.5740) Resonance

\section{INTRODUCTION}

Terahertz (THz) waves, which span the frequencies from $0.1 \mathrm{THz}$ to $10 \mathrm{THz}$, lie in the frequency gap between the infrared and microwave and have received considerable attention during the past decades [1]. One of the most important devices in $\mathrm{THz}$ applications is the bandpass filter, which can be widely applied in imaging, spectroscopy, molecular sensing, security, drug identification, or other systems [2]. Most investigators have focused on the design and fabrication of terahertz filters with a single resonant frequency [3-6]. Nowadays, for spectroscopic applications and astronomical detection, multiple band terahertz bandpass filters are required [7]. On the other hand, the recent trend in terahertz communication systems is to utilize multiple frequency bands and multiple beams to significantly increase the communication capacity [8]. This provides an economical means of developing multi-mission systems and creates a potential demand for multi-band terahertz filters.

Multi-band bandpass filters have gained great attention in wireless communication systems [9-12]. However, in the terahertz frequency range, articles concerning multi or dual-band bandpass filters are still limited. A metal-dielectric dual-band filter consisting of double four-legged loaded slots with different dimensions in one unit cell was investigated theoretically, it was found that the resonant frequencies occurred at 0.183 and $0.22 \mathrm{THz}$ [13], a dual-band terahertz bandpass filter centered at $0.97 \mathrm{THz}$ and $1.37 \mathrm{THz}$ was simulated by exciting multiple resonances of a metal-dielectric-metal structure, both the passbands show high transmissions up to $90 \%$ [14]. Recently, photonic crystal dual-mode dual-band terahertz bandpass filters have been designed. They are formed by a point defect microcavity that is equipped with one large and three smaller auxiliary perturbation rods [15]. A metaldielectric-metal (MDM) sandwich structure consisting of periodic square close ring arrays on both sides of a flexible dielectric substrate has been numerically studied, the designed structure exhibits a multiband transmission with low average insertion loss, steep skirts and high out-of-band rejection [16]. For the terahertz multi-band bandpass filters reported above [13-16], no further experimental demonstration was

\footnotetext{
*Corresponding author: qilimei1204@163.com

Color versions of one or more of the figures in this paper are available online.
} 
carried out.

By perforating metal films with three different geometric shapes, terahertz multi-band filters with strong transmission peaks have been manufactured and measured, and it has also been demonstrated that near-unity transmittance can be obtained at desired terahertz frequencies in plasmonic metamaterials through the shape resonance [17]. By deposition of identical metallic frequency selective surface layers on the upper and lower sides of a quartz crystal to form the metal-dielectric-metal sandwiched structure, second-order bandpass $\mathrm{THz}$ filters have been fabricated [18, 19], however, the fabrication processes of these metal-dielectric-metal filters are relatively complicated compared with the metaldielectric or single-layer metal structure. Multi-band terahertz filters consisting of two and three concentric rings complementary structure have been demonstrated based on metaldielectric structures, results found this structure showed great insensitivity to incidence angles [20].

In this paper, dual-band terahertz filters are designed based on frequency selective surfaces. Three samples are fabricated by perforating two different rectangular holes in one unit with laser in $100 \mu$ m-thick molybdenum. Experimental results measured by a $\mathrm{THz}$ time-domain spectroscopy system show agreement with simulation results. In contrast with most commonly used metal-dielectric structure or metal-dielectric-metal sandwiched structures, the dual-band filters are designed with only one metal layer, which makes fabrication easy and cost low. In addition, the design process is straightforward and simple according to the physical concept and some formulas. Due to the weak coupling between the two rectangular holes in the unit cell, good selectivity performance can be easily achieved both in the lower and higher bands by tuning dimensions of the two slots. Physical mechanisms of the dual-band resonant responses are also clarified.

\section{SIMULATIONS AND DISCUSSIONS}

A Frequency Selective Surface (FSS) is a periodic assembly of one- or two-dimensional resonant structures that exhibits bandpass or bandstop filtering [21-23]. A common class of bandpass FSS can be constructed by placing a periodic array of conducting elements on a dielectric substrate or slots in a conducting surface. Figure 1(a) shows the top

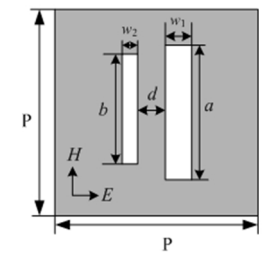

(a)

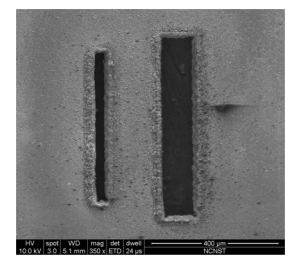

(b)

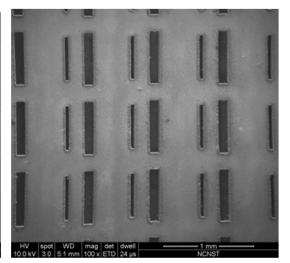

(c)
FIG. 1. (a) Unit cell of the dual-band filter, (b) and (c) Microscope images of one fabricated sample at scale $400 \mu \mathrm{m}$ and $1 \mathrm{~mm}$. view of the unit cell of the dual band FSS filter, the dimensions of the design are $\mathrm{P}=770 \mu \mathrm{m}, \mathrm{a}=590 \mu \mathrm{m}, \mathrm{b}$ $=500 \mu \mathrm{m}, \mathrm{w}_{1}=105 \mu \mathrm{m}, \mathrm{w}_{2}=35 \mu \mathrm{m}$, and $\mathrm{d}=230 \mu \mathrm{m}$. Fig. 1(b) shows the scanning electron micrograph of the fabricated structure. This designed filter can be easily constructed by perforating a periodic array of air slots in $100 \mu \mathrm{m}$-thick molybdenum (conductivity $\sigma=1.76 \times 10^{7} \mathrm{~S} / \mathrm{m}$ ) with a laser (LPKF ProtoMat S43). For the rectangular cell in the frequency selective surface, the resonant frequency can be nearly calculated by the equation [24]:

$$
L=\frac{\lambda}{2}=\frac{c}{2 f \sqrt{\varepsilon_{r}}}
$$

where $L$ is the largest dimension of the rectangular holes, $\lambda$ and $f$ are the resonant wavelength and resonant frequency, respectively. As no dielectric material is introduced in the structure, i.e. $\varepsilon_{\mathrm{r}}=1$, then, the resonant frequency for the long and short rectangular slots in Fig. 1 can be calculated as $\mathrm{f}_{1}=\mathrm{c} / 2 \mathrm{a}=0.254 \mathrm{THz}$ and $\mathrm{f}_{2}=\mathrm{c} / 2 \mathrm{~b}=0.30 \mathrm{THz}$, respectively.

The dual band filter can be modeled as an infinite array in CST Microwave software by utilizing full Floquent mode implementation to simulate a single unit cell of the FSS. For the electric field perpendicular to the long edges of the resonators as stated in Fig. 1(a), the transmission can be obtained from the S-parameters. Three differently configured FSS filters, as shown in the inserts to Fig. 2, are simulated. All of them exhibit band pass characteristics. There is only a single-band in the frequency from $0.1 \mathrm{THz}$ to $0.45 \mathrm{THz}$ both for configuration I or configuration II. For configuration I, the resonant peak occurs at $0.252 \mathrm{THz}$ with the maximum transmission $97 \%$ and the $3 \mathrm{~dB}$ relative bandwidth $14 \%$. For configuration II, the resonant peaks occur at $0.294 \mathrm{THz}$ with the maximum transmission $87 \%$ and the $3 \mathrm{~dB}$ relative bandwidth $4 \%$. The simulated resonant peaks at $0.252 \mathrm{THz}$ and $0.294 \mathrm{THz}$ for the configurations

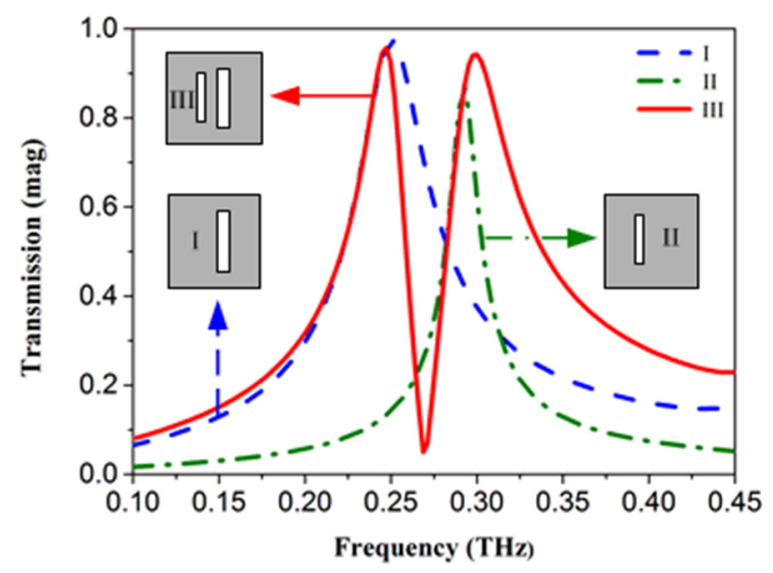

FIG. 2. Numerical simulation results of transmission curves at normal incidence for three different configurations indicated in the insets. 
I and II are in accordance with the theoretical calculation values $0.254 \mathrm{THz}$ and $0.3 \mathrm{THz}$, respectively.

As is shown in configuration III, deploying the two types of resonators (configuration I or configuration II) in one unit cell results in a dual-band, a superposition of configurations I and II. Two resonances occur at 0.247 $\mathrm{THz}$ and $0.3 \mathrm{THz}$, respectively, which are in accordance with the theoretical results $0.254 \mathrm{THz}$ and $0.3 \mathrm{THz}$. For the first band, the maximum transmission is $96 \%$ with the $3 \mathrm{~dB}$ relative bandwidth $9 \%$, and for the second band, the maximum transmission is $94 \%$ with the $3 \mathrm{~dB}$ relative bandwidth $10 \%$. However, the central frequency, the maximum transmission and the $3 \mathrm{~dB}$ relative bandwidth of configuration III do not complete correspond to that of configurations I and II due to the coupling among the neighboring resonators. To further clarify the physical mechanism of the two pass bands, electric field distribution diagrams at the resonant frequencies of $0.247 \mathrm{THz}$ and $0.3 \mathrm{THz}$ are given Fig. 3, it is observed that the electric field resonance occurs in the long rectangular hole at $0.247 \mathrm{THz}$, and the electric field resonance occurs in the short rectangular hole at $0.3 \mathrm{THz}$, Outside the two slots, electric field values are very weak. Consequently, the two pass bands arise from a superposition of two different neighboring holes.

The characteristics of the dual-band filter actually depend on its geometrical parameters. Figure 4 shows the influence of distance $\mathrm{d}$ and period length $\mathrm{p}$ on the transmittance spectra while the remaining parameters are fixed. As shown in Fig. 4(a), on increasing the distance between the two rectangular holes from $70 \mu \mathrm{m}$ to $330 \mu \mathrm{m}$, the left sides of the two transmission bands have no change. Location of the first band shifts to the low frequency with a small decrement of $0.001 \mathrm{THz}$, and location of the second band shifts to the high frequency with an increment of 0.002 THz. Therefore, distance between the holes has small influence on the transmission of the dual-band filter. In Fig. 4(b), as the periodic length varies from $650 \mu \mathrm{m}$ to $750 \mu \mathrm{m}$, bandwidths of the two bands decrease. Location of the first band shifts to the high frequency with an increment of $0.001 \mathrm{THz}$, while location of the second band shifts to the low frequency with a decrement of $0.002 \mathrm{THz}$.

Figure 5 shows the transmittance spectra for various lengths of large hole a and small hole b. As shown in Fig.

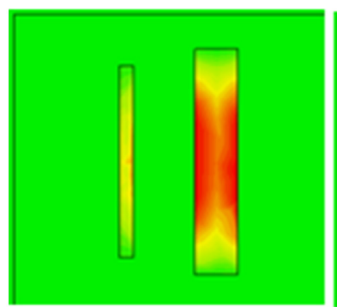

(a)

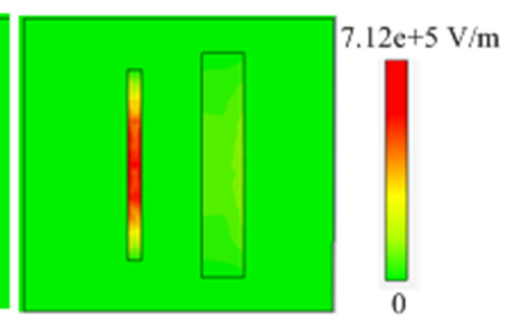

(b)
FIG. 3. Electric field distribution diagrams at the resonant frequencies. (a) $0.247 \mathrm{THz}$ and (b) $0.3 \mathrm{THz}$. 5(a), increasing length of the large hole causes an obvious red shift in the transmission peaks in the first band, while there is little shift in the second band. This result can be explained from the resonance frequency of the first band in Eq. (1). Because the resonance frequency is mainly dependent on size a, i.e., $f_{1}=c / 2 a$. For $a=550,580$ and 610 $\mu \mathrm{m}$, the simulated transmission peaks $f_{1}$ are $0.261,0.251$ and $0.240 \mathrm{THz}$, respectively, which are nearly corresponding with the calculated results of $0.273,0.259$ and $0.246 \mathrm{THz}$, respectively. On the other hand, when slot length a increases, both the peaks and the bandwidths of the first band increase because more waves near the resonant frequency can be transmitted through the filter. In Fig. 5(b), the transmission peaks in the second band decrease with increasing $b$, because the resonance frequency is dependent on size $b$, i.e., $\mathrm{f}_{2}=\mathrm{c} / 2 \mathrm{~b}$. For $\mathrm{b}=480,510$ and $540 \mu \mathrm{m}$, the simulated transmission peaks are $0.307,0.291$ and $0.278 \mathrm{THz}$, respectively, which are again in good agreement with the calculated results of $0.313,0.294$ and $0.278 \mathrm{THz}$, respectively.

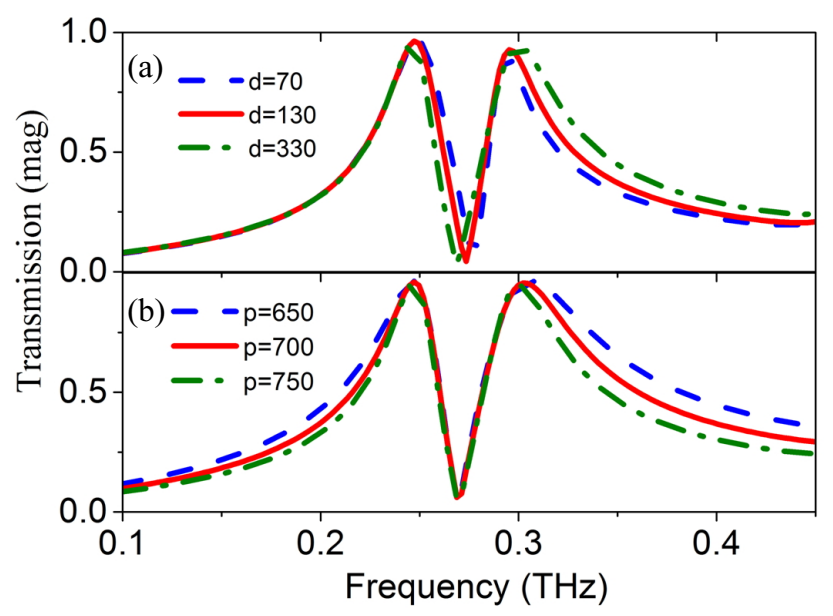

FIG. 4. Transmission of the dual-band filter for different distance and period (a) distance d, and (b) period $\mathrm{p}$.

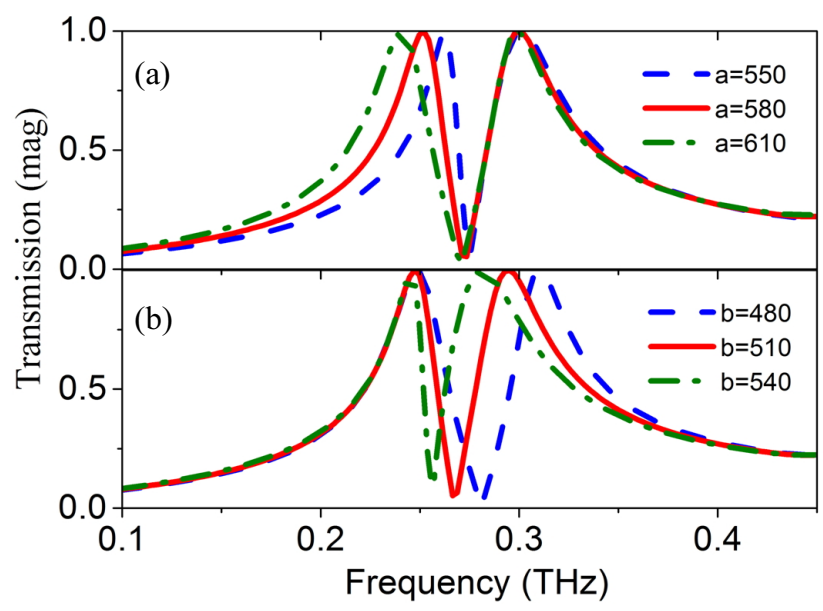

FIG. 5. Transmission of the dual-band filter for different rectangular lengths, (a) large hole a, and (b) small hole b. 


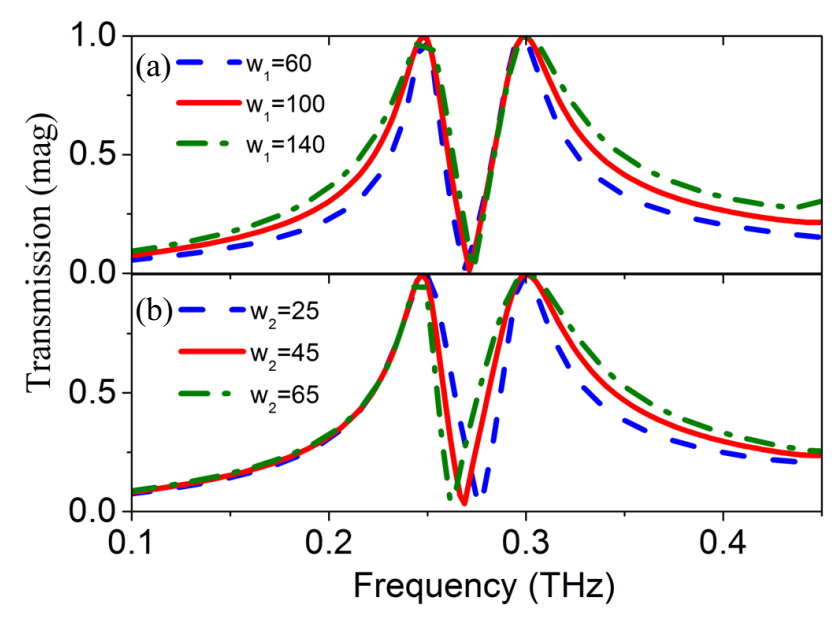

FIG. 6. Transmission of the dual-band filter for different rectangular widths, (a) large hole $\mathrm{w}_{1}$, and (b) small hole $\mathrm{w}_{2}$.

Figure 6 shows the transmittance spectra for various values of the slot widths $\mathrm{w}_{1}$ and $\mathrm{w}_{2}$, while the remaining parameters are fixed. In Fig. 6(a), as width of the large slot $\mathrm{w}_{1}$ increases from 60 to $140 \mu \mathrm{m}$, both the transmission peaks and bandwidths of the two bands increase. In Fig. 6(b), as width of the small slot $w_{2}$ increases from 25 to 65 $\mu \mathrm{m}$, the transmission peaks of the two bands tend to increase. Bandwidth of the second band increases, while bandwidth of the first band decreases. Those results in Fig. 4, Fig. 5 and Fig. 6 confirm that the location and bandwidth of the two bands can be easily tuned by parameters of the filters. Transmission locations of the two bands are mainly determined by the length $a$ and $b$ of the two rectangular holes, while the bandwidths are influenced by all parameters.

\section{EXPERIMENTS AND DISCUSSIONS}

In this work, three samples are fabricated by perforating a $100 \mu \mathrm{m}$-thick molybdenum layer with a periodic array of two rectangular holes with a laser (LPKF ProtoMat S43). Table 1 shows the dimensions of the samples. All the samples have the same period $\mathrm{P}$, width $\mathrm{w}_{1}, \mathrm{w}_{2}$ and distance $\mathrm{d}$, but with different rectangular lengths $\mathrm{a}$ and $\mathrm{b}$. Each fabricated sample has a $25 \times 25$ square array and a very good uniformity was achieved across the $20 \times 20 \mathrm{~mm}$ region. Terahertz time-domain-spectroscopy (THz-TDS) was used to characterize the dual-band responses. The functional frequency region of THz-TDS is $0.05-2.5 \mathrm{THz}$ in this case. Figure 7 shows the diagram of the THz-TDS setup. In brief, the output of a mode-locked Ti-sapphire (Spectra Physics Mai Tai HP), with pulse duration of $70 \mathrm{fs}$, centered wavelength at $800 \mathrm{~nm}$, and repetition rate of $82 \mathrm{MHz}$, is used to generate and detect the terahertz transient. The THz pulses are generated from a low-temperature growth GaAs photoconductive antenna, and a ZnTe crystal with (110) orientation is used as the $\mathrm{THz}$ wave detector. To fix the 100-micronthick Mo filter for testing, a circular aperture is used. Figure
TABLE 1. Geometric parameters of the fabricated dual-band filters samples $(\mu \mathrm{m})$

\begin{tabular}{c|c|c|c|c|c|c}
\hline \hline Parameters & P & $\mathrm{a}$ & $\mathrm{b}$ & $\mathrm{w}_{1}$ & $\mathrm{w}_{2}$ & $\mathrm{~d}$ \\
\hline Sample I & 770 & 590 & 500 & 105 & 35 & 230 \\
\hline Sample II & 770 & 570 & 450 & 105 & 35 & 230 \\
\hline Sample III & 70 & 690 & 485 & 105 & 35 & 230 \\
\hline
\end{tabular}

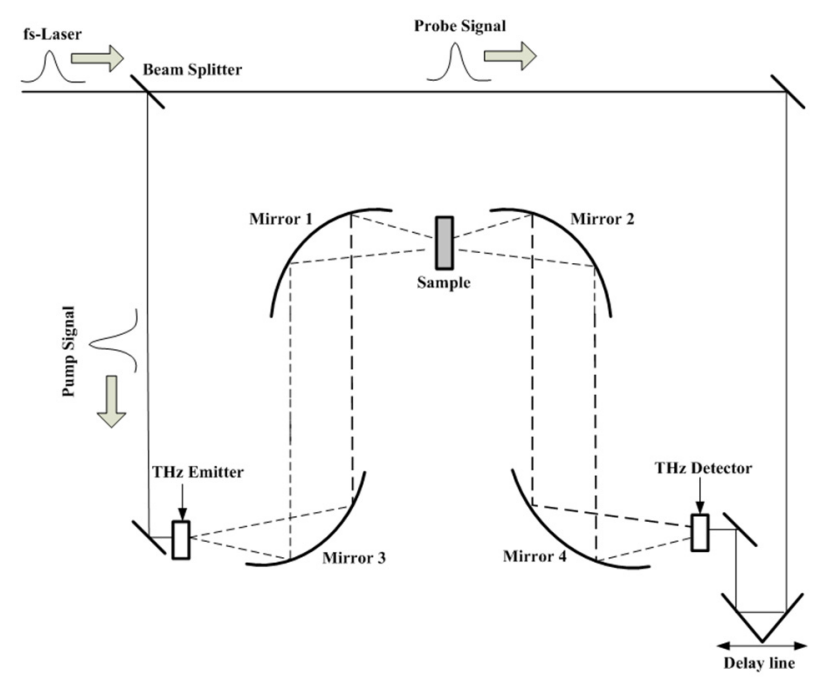

FIG. 7. Diagram of the THz-TDS test system.

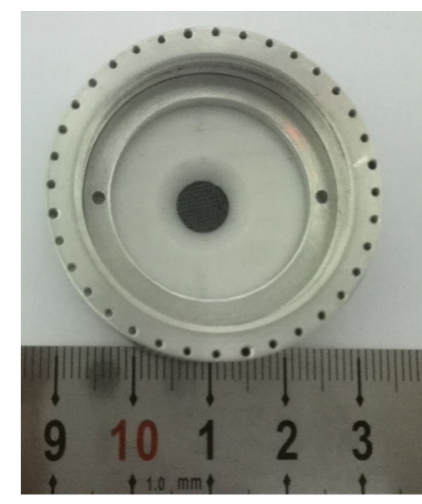

(a)

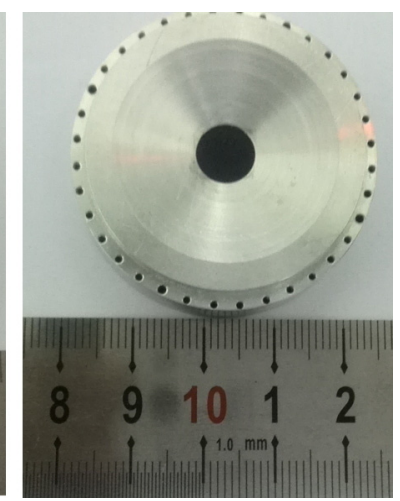

(b)
FIG. 8. The circular aperture to fix the samples. (a) back, (b) front.

8(a) and (b) show the back view and front view, respectively, of the circular aperture used to fix a sample in the center.

Figure 9 shows the $\mathrm{THz}$ wave transmittance characteristics of the three samples at frequencies from 0.1 to $0.45 \mathrm{THz}$, where the solid and dashed lines represent the measured and simulated results, respectively. The measured results of sample I show transmittance peaks of $73 \%$ at $0.245 \mathrm{THz}$ and $71 \%$ at $0.30 \mathrm{THz}$, while the simulated results show transmittance peaks of $100 \%$ and $96 \%$ at $0.249 \mathrm{THz}$ and $0.3 \mathrm{THz}$, respectively. For sample II, the measured results 


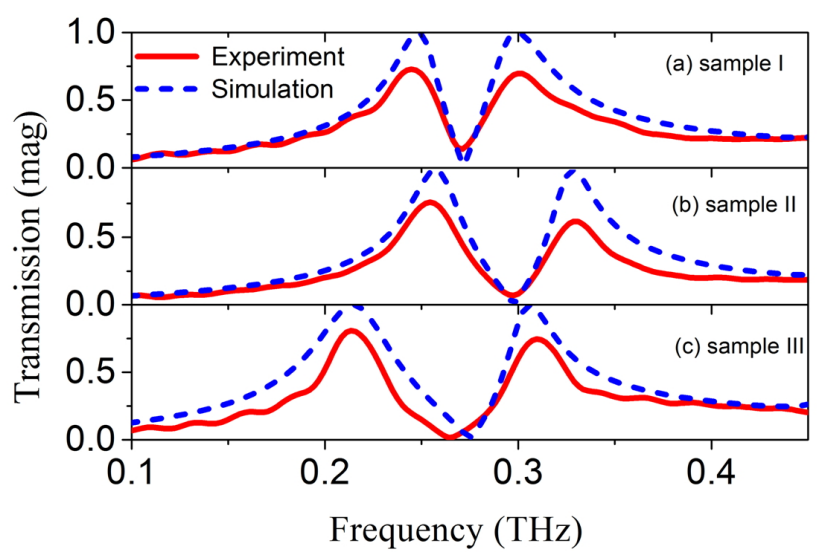

FIG. 9. Experimental transmission spectra of the dual-band filters.

show transmittance peaks of $76 \%$ at $0.249 \mathrm{THz}$ and $63 \%$ at $0.33 \mathrm{THz}$, while the simulated results show transmittance peaks of $97 \%$ at $0.26 \mathrm{THz}$ and $99 \%$ at $0.33 \mathrm{THz}$, respectively. For sample III, the measured results show transmittance peaks of $80 \%$ at $0.214 \mathrm{THz}$ and $75 \%$ at 0.31 $\mathrm{THz}$, while the simulated results show transmittance peaks of $100 \%$ and $96 \%$ at $0.214 \mathrm{THz}$ and $0.3 \mathrm{THz}$, respectively.

The measured transmission curves match the simulated curves, however, there is a slight shift in the resonance frequencies caused by the dimension error processing. On the other hand, the larger loss of the peaks in the measurement may be attributed to the roughness of the fabricated samples. Roughness of the metal can't be set in the CST software. But as we know roughness of metal affects conductivity of the metal. By simulation, we found that the larger the conductivity of the metal, the higher transmission of the filter. Making the samples smooth or using metals with higher conductivity may increase transmission of the two bands. In addition, the periodic boundary conditions approximate an infinite array in the simulations, while the experimental results were measured with limited periods, this discrepancy will also affect the results.

\section{CONCLUSION}

In conclusion, we have designed and fabricated dual-band FSS filters in the terahertz band by perforating two different sizes of rectangular slots with laser in Mo. Experimental measurements demonstrate good agreement with simulation results. These structures have good selectivity performance both in the lower and higher bands by tuning parameters of the two neighboring rectangular holes. Transmission locations of the two bands are mainly determined by the lengths a and $\mathrm{b}$ of the two rectangular holes, while their bandwidths are influenced by all parameters. As the design process is straightforward, the resonant peaks of the two bands can be simply estimated according to their physical concepts and the corresponding formulas. Finally, the designed single-layer frequency selective surface filters in the terahertz band also have great advantages of easy fabrication and low cost. The encouraging results afforded by this designed filter could find its applications in dual-band sensors, $\mathrm{THz}$ communication systems and other emerging $\mathrm{THz}$ technologies.

\section{ACKNOWLEDGMENT}

This work was supported by the National Natural Science Foundation of China (Nos. 11174280, 61107030), the Knowledge Innovation Program of Chinese Academy of Sciences (No. YYYJ-1123) and the China Postdoctoral Science Foundation.

\section{REFERENCES}

1. B. Ferguson and X. Zhang, "Materials for terahertz science and technology," Nature Materials 1, 26-33 (2002).

2. H. T. Chen, W. J. Padilla, J. M. O. Zide, A. C. Gossard, A. J. Taylor, and R. D. Averitt, "Active metamaterial terahertz devices," Nature 444, 597-600 (2006).

3. R. Dickie, R. Cahill, H. S. Gamble, V. F. Fusco, P. G. Huggard, B. Moyna, M. Oldfield, N. Grant, and P. Maagt, "Polarisation independent bandpass FSS," Electron. Lett. 43, 1013-1015 (2007).

4. R. Dickie, R. Cahill, V. F. Fusco, H. S. Gamble, and N. Mitchell, "THz frequency selective surface filters for earth observation remote sensing instruments," IEEE Trans. Terahertz Science and Technology 1, 450-461 (2011).

5. N. Jin and J. Li, "Terahertz wave bandpass filter based on metamaterials," Microwave and Optical Technology Letters 53, 1858-1860 (2011).

6. Y. Zhu, S. Vegesna, V. Kuryatkov, M. Holtz, M. Saed, and A. A. Bernussi, "Terahertz bandpass filters using doublestacked metamaterial layers," Opt. Lett. 37, 296-298 (2012).

7. M. Tarenghi, "The atacama large millimeter/submillimeter array: overview \& status," Astrophysics and Space Science 313, 1-7 (2008).

8. H. J. Song and T. Nagatsuma, "Present and future of terahertz communications," IEEE Transactions on Terahertz Science and Technology 1, 256-263 (2011).

9. H. K. Jhuang, C. H. Lee, and C. I. G. Hsu, "Design of compact microstrip dual-band bandpass filters with $\lambda / 4$ stepped-impedance resonators," Microwave and Optical Technology Letters 49, 164-168 (2007).

10. Y. Yang, X. Wang, and H. Zhou, "Dual-band frequency selective surface with miniaturized element in low frequencies," Progress In Electromagnetics Research Letters 33, 167-175 (2012).

11. G. Chaudhary, Y. Jeong, K. Kim, and D. Ahn, "Design of dual-band bandpass filters with controllable bandwidths using new mapping function," Progress In Electromagnetics Research 124, 17-34 (2012).

12. H. Liu, Y. Fan, Z. Zhang, Y. Zhao, W. Xu, X. Guan, L. Sun, and Y. He, "Dual-band superconducting bandpass filter using embedded split ring resonator," IEEE Transactions 
on Applied Superconductivity 23, 1300304 (2013).

13. C. Guo, H. Sun, and X. Lu, "A novel dualband frequency selective surface with periodic cell perturbation," Progress In Electromagnetics Research B 9, 137-149 (2008).

14. Y. J. Chiang and T. J. Yen, "A high-transmission dualband terahertz bandpass filter by exciting multiresonance of metamaterials," Proc. SPIE 8070, Metamaterials VI, 80700V (2011).

15. O. Karakilinc and M. Dinley, "Design of dual-mode dual-band photonic crystal bandpass filters for terahertz communication applications," Microwave and Optical Technology Letters 57, 1806-1810 (2015).

16. X. Chen and W. Fan, "A multiband $\mathrm{THz}$ bandpass filter based on multiple-resonance excitation of a composite metamaterial," Materials Research Express 2, 055801 (2015).

17. J. W. Lee, M. A. Seo, D. J. Park, D. S. Kim, S. C. Jeoung, Ch. Lienau, Q.-H. Park, and P. C. M. Planken, "Shape resonance omni-directional terahertz filters with near-unity transmittance," Opt. Express 14, 1253-1259 (2006).

18. M. Lu, W. Li, and E. R. Brown, "Second-order bandpass $\mathrm{THz}$ filter achieved by multilayer complementary metamaterial structures," Opt. Lett. 36, 1071-1073 (2011).
19. F. Lan, Z. Yang, L. Qi, X. Gao, and Z. Shi, "Terahertz dual-resonance bandpass filter using bilayer reformative complementary metamaterial structures," Opt. Lett. 39, 1709-1712 (2014).

20. L. Qi and C. Li, "Multi-band terahertz filter with independence to polarization and insensitivity to incidence angles," Journal of Infrared, Millimeter, and Terahertz Waves 36, 1137-1144 (2015).

21. X. Zhang, Z. Zheng, X. Li, R. Tan, Z. Zhang, Y. Zhou, J. Sun, B. Zhang, and H. Qin, "Terahertz filter based on frequency selective surfaces," Advanced Materials Research 571, 362-366 (2012).

22. C. Lee, W Shim, Y. Moon, and C. Seo, "Design of ultra-wide band-pass filter based on metamaterials applicable to microwave photonics," J. Opt. Soc. Korea 16, 288-291 (2012).

23. J. W. Lee, M. A. Seo, D. H. Kang, K. S. Khim, S. C. Jeoung, and D. S. Kim, "Terahertz electromagnetic wave transmission through random arrays of single rectangular holes and slits in thin metallic sheets," Physical Review Letters 99, 137401 (2007).

24. B. A. Munk, Frequency Selective Surfaces: Theory and Design (John Wiley and Sons Inc., 2000), p. 393. 